\title{
Management of epilepsy in the elderly
}

\author{
Juan José Poza \\ Department of Neurology, Hospital \\ Donostia, San Sebastián, Spain
}

\begin{abstract}
Epilepsy among the elderly is a frequently occurring pathology, differing in etiology, clinical presentation and prognosis from those of young people. In addition, beyond a certain age, physiological modifications are produced in the metabolism which alter the pharmacokinetics of antiepileptic drugs (AEDs), increasing the risk of pharmacological interactions, already greater in these patients due to the frequency of poly-pharmacy. Furthermore, elderly patients are particularly sensitive to certain secondary effects of AEDs, as for example, cognitive disturbances, osteoporosis or weight increase. Given that the efficacy of the major AEDs is a priori quite similar, and that the epilepsies occurring in this age-group generally have a good prognosis, the selection of an AED will depend more upon its pharmacokinetics and ability to induce certain secondary effects than on its efficacy. In this respect, levetiracetam and pregabalin, followed by oxcarbazepine and lamotrigine have the most favorable pharmocokinetical profile. Moreover, on the whole these drugs have very few cognitive effects, do not induce osteoporosis and, with the exception of pregabalin, do not affect weight, making them the first selection for use in the treatment of epilepsy in the elderly.
\end{abstract}

Keywords: epilepsy, the elderly, antiepileptic drugs

\section{Introduction}

Epilepsy is particularly common in the over 60s. A number of epidemiological studies carried out around the world have shown that the prevalence of epilepsy rises rapidly above this age, even increasing beyond the other prevalence peak which appears in infancy. On the one hand, we have those patients whose epilepsy started at whatever age and who are now over 60 year-old, and on the other hand those in whom it started after this age, the latter representing $25 \%$ of the new cases of epilepsy (Sander and Shoevon 1996). The incidence of epilepsies in the over 60s is double the figure for those aged between 40 and 59 years old and follows a lineal growth reaching a figure of 140/100,000 for the over 80s, three times the figure for those in the 60-69 year-old age group (Loiseau et al 1990; Tallis et al 1991; Hauser 1992; Hauser et al 1993). Thus, epilepsy together with cerebrovascular pathology and dementias is one of the most common neurological pathologies in old age. Moreover, as will be seen below, both cerebrovascular pathology and dementias may be a cause of epilepsy. Given the tendency towards an ageing population in the West, an increase in the number of epileptic patients, particularly in this segment of the population, can be expected.

In spite of the fact that more and more often, the epileptic patients encountered in the surgery are over 60 years old, this age group is rarely represented in the clinical trials which have led to the approval of the different antiepileptic drugs (AEDs). The high co-morbidity, the frequency of polypharmacy, the increased risk of secondary effects and of mortality due to intercurrent causes in these patients are, among others, the reasons for their almost systematic exclusion from clinical trials. Therefore, given the lack of evidence based on clinical trials, we shall try to determine the most appropriate antiepileptic drugs for these patients according to the characteristics of their epilepsy, the modifications in the metabolic capacity of the drugs which arise with age, 
the possibility of secondary effects of special relevance to the elderly and the characteristics of the different drugs.

\section{Characteristics of epilepsy in the elderly}

In elderly patients, the critical phenomenology presents peculiarities which may make diagnosis more difficult. For example, automatisms and auras are unusual in the elderly and often the crises present themselves as episodes of confusion or syncopal events. Therefore, in almost half of the elderly patients who are finally diagnosed with epilepsy, epilepsy is not the initial suspected diagnosis (Ramsay et al 2004). Furthermore, the appearance of an epileptic status as a form of presentation of the epilepsy is also more common among the elderly (Pryor et al 2002).

It should be remembered that the elderly are more at risk of presenting reactive crises. The metabolic disorders which may provoke a crisis, including hypo- or hyperglycemia, uremia, hyponatremia or hypocalcaemia, are more common among the elderly. In addition, this section of the population tends to use drugs more frequently, including drugs potentially capable of inducing an epileptic crisis, as are some antidepressants, neuroleptics and certain antibiotics.

Furthermore, the incidence of epilepsy is particularly high among the over $60 \mathrm{~s}$. However, this increase in the incident rate only corresponds to certain types of epilepsy. Almost no idiopathic epilepsies start in this age group. In any case, we may encounter patients with idiopathic epilepsies which started at much earlier ages and who have reached old age. Therefore, the possibility of a generalized epilepsy commencing in old age is remote, and given an patient aged over 60 who has developed epilepsy we should consider a focal epilepsy even when there is no clear focal start to the crises (Hiyoshi and Yagi 2000).

Practically two thirds of epilepsies which develop at this age are symptomatic, and the other third are cryptogenic. The most common cause of epilepsy among the over 60 s is the cerebralvascular pathology, related to almost $50 \%$ of epilepsies among the elderly (Asconape and Penry 1991). Moreover, epilepsy may be the first sign of an underlying vascular pathology which has not yet surfaced. Thus, the risk of epilepsy in hypertensive patients is five times higher than in those who are not hypertensive, probably in relation to a pathology of small blood vessels causing irritating symptoms before provoking an ictus ( $\mathrm{Ng}$ et al 1993). Cleary et al monitored a cohort of 4,709 patients with cryptogenic epilepsy which started at over 60 years old, and found that the relative risk of suffering an ictus in these patients compared with controls from the same population was 2.89 , suggesting that in many of these patients there was an alteration to the cerebral blood flow which manifested itself first as an epilepsy and then as an ictus (Cleary et al 2004).

Other frequently related causes of epilepsy in the elderly are brain tumors, cranial trauma and dementia.

The fact that we encounter symptomatic epilepsies conditions the therapeutic attitude, as the risk of recurrence is very high (Bergey 2004), over 90\%, and therefore starting treatment after the first crisis can be considered. In the majority of cases, good control of these epilepsies can be expected, but treatment will be indefinite, and the suspension of treatment even after several years without a crisis can not be considered, as recurrence is almost certain. However this should be assessed for each particular case, as it will largely depend upon the risks that the patient and the doctor are willing to assume (Serrano Castro, Ramos Lizana et al 2005).

\section{Modifications of the metabolism in the elderly}

The physiology of elderly patients presents alterations which modify the pharmacokinetics of AEDs, making patients more susceptible to secondary effects. Firstly, there is an atrophy of the gastric mucosa and a reduction in the intestinal motility, which may modify the absorption of AEDs making it irregular. In addition, there is a reduction in total body water, leading to a modification of the volume of distribution of the drugs and their half life, which may on occasions have significant consequences. Thus, the half life of Diazepam goes from 20 hours in young people to 80 hours in those individuals aged over 80 , with the resulting risk of a build-up of the drug.

The bonding of drugs to plasmatic proteins is also reduced. As a result, the free fraction of drugs which bond well to proteins, such as valproic acid, phenitoin or tiagabine may increase by as much as $60 \%$, bringing with this the risk of secondary effects due to intoxication (Serrano Castro, Casado-Chocan et al 2005).

Finally, the capacity to eliminate drugs is also lower in the elderly than in young people. The hepatic metabolism is much slower, which may lead to a build-up of drugs with intense hepatic metabolism, such as carbamazepine, phenitoin or lamotrigine. Moreover, these patients will have a higher sensitivity to phenomenons of hepatic induction or inhibition, given that their hepatic reserve is reduced. In addition, glomerular filtration is also reduced, which must be remembered when prescribing renally eliminated drugs such as levetiracetam, gabapentin or pregabalin.

An added problem is that the elderly often take multiple medications, increasing the risk of interactions and the 
possibility of secondary effects. In this respect, the lower the bonding to proteins and the lower the hepatic metabolism of a drug, the easier it will be to use and the lower the risk of interaction with other possible concomitant drugs.

\section{Secondary effects of special relevance in the elderly}

In general, elderly patients are more sensitive to the secondary effects of drugs, even at reduced doses. In addition, certain secondary effects frequently related to AEDs are of special relevance to the elderly.

Due to the inhibiting action on the central nervous system, many AEDs may cause cognitive disturbances. Elderly patients often present a reduction in their cerebral reserve capacity, making them particularly sensitive to this secondary effect. The possibility of inducing cognitive disturbances is very high with drugs such as phenobarbital, primadone, or the benzodiazepines, and is more moderate with phenitoin and topiramate. The last in the list often causes a characteristic difficulty in finding words. Drugs less frequently causing cognitive disturbances, and which are therefore safer for use by this segment of the population include oxcarbazepine, levetiracetam, gabapentin and lamotrigine.

Osteoporosis is a common disorder among the over $60 \mathrm{~s}$, particularly among women, and may be the cause of significant disability due to chronic pain and a predisposition to fractures. Various AEDs may induce osteoporosis, emphasizing a risk which is already high merely as a result of ageing. Some, such as phenobarbital, phenitoin or carbamazepine, do so by interfering with the metabolism as vitamin D. Valproate can also induce osteoporosis by activating the osteoblasts which destroy bone tissue. The risk of complications due to osteoporosis is multiplied in an elderly patient if we add in an AED capable of inducing osteoporosis. Therefore, the risk of hip fractures in women over 65 is doubled when taking one of these drugs (Ensrud et al 2004). As such, a priori, we should avoid the use of osteoporosis inducing drugs in this segment of the population.

The possibility of various AEDs causing an increase in weight is well known. In general, we tend to consider this secondary effect when treating young patients, especially women, based on the assumption that these patients may be most concerned about their figure. However, it is extremely important to consider the possibility of causing an increase in weight in elderly patients, not for aesthetic reasons but for reasons of health. An increase in weight increases the risk of type II diabetes, cardiovascular mortality and osteoarticular problems in a population where the risk is already higher. Moreover, an increase in weight leads to clumsiness in a population which tends to be clumsy, and increases the risk of falls in a population with an increased risk of osteoporosis, and therefore, greater risk of fractures. The ADFs most frequently associated to weight increase include Valproate (more than half the patients), carbamazepine (approximately a quarter of patients), gabapentin (15\%-20\%) and pregabalin (14\%) (De Toledo et al 1997; Easter et al 1997; Biton 2003; Arroyo et al 2004). Topiramate may induce weight loss, which can be useful for overweight patients, but in other cases this effect may also be harmful and lead to malnutrition (Ben-Menachem et al 2003). Therefore, the most recommended drugs for this segment of the population are those which do not tend to modify weight, including lamotrigine, levetiracetam and oxcarbazepine (Devinsky et al 2000; Gidal, Sheth, Magnus et al 2003).

\section{First choice AEDs for the elderly}

From the above, we can deduce that we are faced with a population which will frequently present symptomatic focal epilepsies, for which a good response to medical treatment can be expected, even at lower dosages (Kwan and Brodie 2001). The majority of AEDs available have demonstrated a similar efficacy for treating this type of crisis (Mattson et al 1985, 1992; Brodie et al 1999, 2002). Therefore, the choice of drug will fundamentally depend upon its pharmacokinetic profile and the likelihood of causing secondary effects (Sánchez-Alvarez et al 2005) which, as we have seen, have particular repercussions among the elderly. Therefore we have analyzed the AEDs from this perspective.

Phenobarbital is a powerful antiepileptic, but its significant cognitive effects, the possibility of inducing osteoporosis and possible pharmacological interactions rule it out for the treatment of epilepsies developing for the first time among this group of patients.

Phenytoin is probably the most widely used drug among the elderly due to the possibility of a rapid titration and intravenous administration. However, the complexity of its pharmacokinetics, the possibility of interaction with other drugs, of inducing osteoporosis and its cognitive effects make it unadvisable (Birnbaum et al 2003).

Valproic acid is an antiepileptic with a broad spectrum, being very efficient for both focal and generalized epilepsies, although we are principally concerned with the former among this population. It can be administered intravenously, enabling rapid titration, the treatment of status epileptics and can be prescribed without problem where the patient is required to follow a strict diet due to an intercurrent disease. The pharmacokinetics present certain problems, including its high 
Table I Pharmacokinetical modifications in the elderly

\begin{tabular}{ll}
\hline Alteration & Cause \\
\hline Reduced absorption & $\begin{array}{l}\text { Atrophy of gastric mucosa } \\
\text { Reduction in intestinal motility }\end{array}$ \\
Alteration in the volume & Reduction in total body water \\
of distribution & Reduction in bonding with proteins \\
Reduced elimination & Slowing down of hepatic metabolism \\
Pharmacokinetic competition & Reduction of glomerular filtration \\
\hline
\end{tabular}

bonding to proteins and its capacity for hepatic inhibition, which may provoke pharmacological interactions. But the main problem lies in the frequency with which it causes weight gain, which, as we have seen, is of particular significance to health among these patients. Other secondary effects of significance among elderly patients include osteoporosis and tremor, and even Parkinson's, in a population particularly exposed to suffering from osteoporosis and tremors or Parkinson's due to their age. Thrombopenia is a secondary effect which also appears more often among the elderly.

Carbamazepine is a powerful antiepileptic, with minimum cognitive effects. Its main disadvantage is its hepatic capacity, making the risk of interaction with other drugs high, of particular significance in a population where polypharmacy is common. In addition it may induce osteoporosis and weight gain. Another two side effects to consider due to their higher frequency among the elderly are hyponatremia and alterations to the heart conduction system, which may cause confused or syncopal episodes respectively.

The problems presented by carbamazepine have largely been resolved with oxcarbazepine, whose capacity for hepatic induction and alterations to the heart conduction system are lower than its predecessor. However, it induces hyponatremia more frequently than carbamazepine does (Kutluay et al 2003).

Topiramate is a powerful antiepileptic but the frequency with which it causes cognitive disorders, especially naming defects makes it unadvisable as a first choice for this population. Other secondary effects to consider given their possible gravity, although uncommon, include renal lithiasis and metabolic acidosis. Finally, the frequency of secondary effects requires a very slow titration of the drug, implying a long lapse in time until the patient is protected (Reife et al 2000).

Lamotrigine is a powerful antiepileptic without cognitive effects, with linear kinetics, making it highly suitable for this population. From a pharmacokinetics perspective, the main disadvantage is its hepatic metabolism, which may be induced by other drugs, with a significant reduction in plasmatic levels (Anderson et al 2002). On the contrary, valproic acid significantly raises its levels and may double its half life (Gidal, Sheth, Parnell et al 2003). In addition it is a slight hepatic inducer. Moreover, the possibility of serious allergic reactions makes very slow titration necessary, taking several weeks for an efficient dose to be reached. This time period is usually lower among elderly patients, as, in general, lower doses are required to control their epilepsy.

Tiagabine is a GABA-ergic drug with little antiepileptic power, requiring administration in three doses, and which often induces significant somnolence, and therefore it is not recommendable for this population.

Gabapentin has a most favorable pharmacokinetical profile, but its low antiepileptic activity makes it unadvisable. Its successor, pregabalin, has the same pharmacokinetic profile, with the advantage that its antiepileptic power is similar to that of other greater AEDs (Gil-Nagel and Gomez Alonso 2005). Treatment can be started immediately with an efficient dosage. The main disadvantages are its capacity to induce somnolence and weight gain (French et al 2003; Brodie 2004).

Finally, levetiracetam is a powerful antiepileptic with a highly favorable pharmacokinetic profile, as it does not bond with proteins, nor does it metabolize in the liver and neither induces nor is induced. It titrates rapidly, and therefore from the start of treatment the patient has an efficient dosage. The only disadvantage is its possibility of provoking slight irritability or psychiatric alterations in under $7 \%$ of elderly patients, usually in patients with prior psychomotor retardation (Ferrendelli et al 2003; Morrell et al 2003; White et al 2003).

Table 1 summarizes the main advantages and disadvantages of each of the AEDs.

Table 2 Secondary effects of special relevance in the elderly

\begin{tabular}{ll}
\hline Secondary effect & AEDs which may cause this effect \\
\hline Cognitive disorder & PB \\
& PRM \\
BDZ \\
PHT \\
TPM \\
Osteoporosis & PB \\
& PHT \\
& CBZ \\
Weight gain & VPA \\
& VPA \\
& CBZ \\
& GBP \\
\hline
\end{tabular}


Table 3 Advantages and disadvantages of each AED

\begin{tabular}{|c|c|c|}
\hline AED & Advantage & Disadvantage \\
\hline \multirow[t]{2}{*}{ Phenobarbital } & Powerful antiepileptic & Marked cognitive effects Osteoporosis \\
\hline & $\begin{array}{l}\text { Possibility of parenteral } \\
\text { administration }\end{array}$ & Many pharmacological interactions \\
\hline \multirow[t]{5}{*}{ Phenytoin } & Powerful antiepileptic & Absorption affected by food or antacids \\
\hline & Possibility of parenteral & High bonding to proteins $(80 \%-95 \%)$ \\
\hline & administration & Complex, non linear kinetics \\
\hline & Rapid titration & Enzymatic inducer. Inducible \\
\hline & Does not affect weight & Osteoporosis \\
\hline \multirow[t]{6}{*}{ Valproic acid } & Powerful antiepileptic & High bonding to proteins (80\%-95\%) \\
\hline & Possibility of parenteral & Enzymatic inhibitor \\
\hline & administration & Weight gain $(>50 \%)$ \\
\hline & Minimum cognitive effects & Osteoporosis \\
\hline & & Tremor \\
\hline & & Thrombopenia \\
\hline \multirow[t]{9}{*}{ Carbamazepine } & Powerful antiepileptic & High bonding to proteins ( $80 \%)$ \\
\hline & Minimum cognitive effects & Hepatic metabolism \\
\hline & & Enzymatic inducer.Auto-induction \\
\hline & & Osteoporosis \\
\hline & & Weight gain \\
\hline & & Hyponatremia \\
\hline & & Alterations to heart conduction system \\
\hline & & Slow titration \\
\hline & & Allergic reaction \\
\hline \multirow[t]{4}{*}{ Oxcarbazepine } & Powerful antiepileptic & Slight hepatic inducer \\
\hline & Linear kinetics & Bonds to proteins $(67 \%)$ \\
\hline & No cognitive effects & Hyponatremia \\
\hline & Does not affect weight & Alteration to heart conduction system \\
\hline \multirow{5}{*}{ Topiramate } & Powerful antiepileptic & Cognitive disorder \\
\hline & Does not bond to proteins & Slight hepatic inducer Inducible \\
\hline & & Renal lithiasis \\
\hline & & Metabolic acidosis \\
\hline & & Slow titration \\
\hline \multirow[t]{5}{*}{ Lamotrigine } & Powerful antiepileptic & Hepatic metabolism \\
\hline & Linear kinetics & Slight hepatic inducer Very inducible \\
\hline & No cognitive effects & Bonds to proteins $(55 \%-60 \%)$ \\
\hline & Does not affect weight & Allergic reactions \\
\hline & & Slow titration \\
\hline \multirow[t]{5}{*}{ Tiagabin } & & Weak antiepileptic \\
\hline & & Three doses \\
\hline & & High bonding to proteins (96\%) \\
\hline & & Hepatic metabolism Inducible \\
\hline & & Somnolence \\
\hline \multirow[t]{4}{*}{ Gabapentin } & Rapid titration & Weak antiepileptic \\
\hline & No hepatic metabolism & Three doses \\
\hline & Does not bond to proteins & Saturable absorption \\
\hline & No interactions & Weight gain \\
\hline \multirow[t]{6}{*}{ Pregabalin } & Powerful antiepileptic & Somnolence \\
\hline & Rapid titration & Weight gain \\
\hline & Rapid absorption not saturable & \\
\hline & No hepatic metabolism & \\
\hline & Does not bond to proteins & \\
\hline & No interactions & \\
\hline \multirow[t]{6}{*}{ Levetiracetam } & Powerful antiepileptic & Uncommon irritability and psychiatric disorders $(<7 \%)$ \\
\hline & Rapid titration & \\
\hline & No hepatic metabolism & \\
\hline & Does not bond to proteins & \\
\hline & No interactions & \\
\hline & No cognitive alteration & \\
\hline
\end{tabular}


In view of these characteristics, we could say that, for a similar a priori efficacy, the drugs with the most favorable pharmacokinetic profile are levetiracetam and pregabalin, followed by oxcarbazepine and lamotrigine, whose hepatic metabolism and bonding to proteins slightly increases the risk of drug interactions. Moreover, these drugs have few cognitive effects, do not cause osteoporosis and, except for pregabalin, do not affect bodyweight, making them particularly attractive for treating epilepsies in the elderly.

\section{Conclusions}

Epilepsy is a common disorder among the elderly. The majority of epileptic patients are over 60 years old. These patients usually have partial symptomatic crises, which tend to reoccur, but which respond well to antiepileptic treatment. Given that a priori the efficacy of all the major AEDs is similar in the treatment of partial symptomatic crises, the choice of AED for use in this population will depend upon the pharmacokinetic properties and side effects. In this respect, the AEDs with a more favorable profile for starting antiepileptic treatment in elderly patients include levetiracetam, pregabalin, lamotrigine and oxcarbazepine.

\section{References}

Anderson GD, Gidal BE, Messenheimer JA, et al. 2002. Time course of lamotrigine deinduction: impact of step-wise withdrawal of carbamazepine or phenytoin. Epilepsy Res, 49:211-7.

Arroyo S, Anhut H, Kugler AR, et al: for the Pregabalin 1008-011 International Study Group. 2004. Pregabalin add-on treatment: a randomized, double-blind, placebo-controlled, dose-response study in adults with partial seizures. Epilepsia, 45:20-7.

Asconape JJ, Penry JK. 1991. Poststroke seizures in the elderly. Clin Geriatr Med, 7:483-92.

Ben-Menachem E, Axelsen M, Johanson EH, et al. 2003. Predictors of weight loss in adults with topiramate-treated epilepsy. Obes Res, 11:556-62.

Bergey GK. 2004. Inicial treatment of epilepsy. Special issues in treating the eldery. Neurology, 63:S40-8.

BirnbaumA, Hardie NA, Leppik IE, et al. 2003. Variability of total phenytoin serum concentrations within elderly nursing home residents. Neurology, 60:555-9.

Biton V. 2003. Effect of antiepileptic drugs on bodyweight: overview and clinical implications for the treatment of epilepsy. CNS Drugs, 17:781-91.

Brodie MJ. 2004. Pregabalin as adjunctive therapy for partial seizures. Epilepsia, 45:Suppl 6:19-27.

Brodie MJ, Chadwick DW, Anhut H, et al. 2002. Gabapentin versus lamotrigine monotherapy: a double-blind comparison in newly diagnosed epilepsy. Epilepsia, 43:993-1000.

Brodie MJ, Overstall PW, Giorgi L. 1999. Multicentre, double-blind, randomised comparison between lamotrigine and carbamazepine in elderly patients with newly diagnosed epilepsy. The UK Lamotrigine Elderly Study Group. Epilepsy Res, 37:81-7.

Cleary P, Shorvon S, Tallis R. 2004. Late-onset seizures as a predictor of subsequent stroke. Lancet, 363:1184-6.

De Toledo JC, Toledo C, De Cerce J, et al. 1997. Changes in body weight with chronic, high-dose gabapentin therapy. Ther Drug Monit, 19:394-6.

Devinsky O, Vuong A, Hammer A, et al. 2000. Stable weight during lamotrigina therapy: a review of 32 studies. Neurology, 54:973-5.

Easter D, O’Bryan-Tear CG, Verity C. 1997. Weight gain with valproate or carbamazepine-a reapprisal. Seizure, 6:121-5.
Ensrud KE, Walczak TS, Blackwell T, et al. 2004. Antiepileptic drug use increases rates of bone loss in older women: a prospective study. Neurology, 62:2051-7.

Ferrendelli JA, French J, Leppik I, et al. 2003. Use of levetiracetam in a population of patients aged 65 years and older: a subset análisis of the KEEPER trial. Epilepsy Behav, 4:702-9.

French JA, Kugler AR, Robbins JL, et al. 2003. Dose-response trial of pregabaline adjunctive therapy in patients with partial seizures. Neurology, 60:1631-7.

Gidal BE, Sheth RD, Magnus L, et al. 2003. Levetiracetam does not alter body weight: analysis of randomized, controlled clinical trials. Epilepsy Res, 56:121-6.

Gidal BE, Sheth R, Parnell J, et al. 2003. Evaluation of VPA dose and concentration effects on lamotrigine pharmacokinetics: implications for conversion to lamotrigine monotherapy. Epilepsy Res, 57:85-93.

Gil-Nagel A, Gómez Alonso J. 2005. Experiencia clínica de la pregabalina en el tratamiento de las epilepsias focales. Rev Neurol, 40:609-13.

Hauser WA. 1992. Seizure disorders: the changes with age. Epilepsia, 33(Suppl 4):S6-14.

Hauser WA, Annegers JF, Kurland LT. 1993. Incidence of epilepsy and unprovoked seizures in Rochester, Minnesota: 1935-1984. Epilepsia, 34:453-68.

Hiyoshi T, Yagi K. 2000. Epilepsy in the elderly. Epilepsia, 41(Suppl 9):31-5.

Kutluay E, McCague K, D'Souza J, et al. 2003. Safety and tolerability of oxcarbazepine in elderly patients with epilepsy. Epilepsy Behav, 4:175-80.

Kwan P, Brodie MJ. 2001. Effectiveness of first antiepileptic drug. Epilepsia, 42:1255-60.

Loiseau J, Loiseau P, Duche B, et al. 1990. A survey of epileptic disorders in elderly patients. Ann Neurol, 27:232-7.

Mattson RH, Cramer JA, Collins JF, et al. 1985. Comparison of carbamazepine, phenobarbital, phenytoin and primidone in partial and secondarily generalized tonic-clonic seizures. N Engl J Med, 313:145-51.

Mattson RH, Cramer JA, Collins JF. 1992. A comparison of valproate with carbamazepine for the treatment of complex partial seizures and secondarily generalized tonic-clonic seizures in adults. The Department of Veterans Affairs Epilepsy Cooperative Study N 264 Group. NEngl $J$ Med, 327:765-71.

Morrell MJ, Leppik I, French J, et al. 2003. The KEEPER trial levetiracetam adjunctive treatment of partial-onset seizures in an open label community-based study. Epilepsy Res, 54:153-61.

Ng SK, Hauser WA, Brust JC, et al. 1993. Hypertension and risk of newonset unprovoked seizures. Neurology, 43:425-8.

Pryor FM, Ramsay RE, Rowan AJ. 2002. Epilepsy in older adults: update from VA Cooperative Study \#428. Epilepsia, 43(Suppl 7):165-6.

Ramsay RE, Rowan AJ, Pryor FM. 2004. Special considerations in treating the elderly patients with epilepsy. Neurology, 62:S24-9.

Reife R, Pledger G, Wu SC. 2000. Topiramate as add-on therapy: pooled analysis of randomized controlled trials in adults. Epilepsia, 41(Suppl 1):S66-71.

Sánchez-Álvarez JC, Galán-Barranco JM, Camino-León R, et al. 2005. Guía terapéutica en epilepsia de la Sociedad Andaluza de Epilepsia 2005; II. Terapéutica antiepiléptica crónica en el adulto y en el niño. Rev Neurol, 40:619-26.

Sander JWAS, Shoevon SD. 1996. Epidemiology of the epilepsies. J Neurol Neurosurg Psychiatry, 61:433-43.

Serrano Castro PJ, Casado-Chocan JL, Mercadé-Cerdá JM, et al. 2005. Guía terapéutica en epilepsia de la Sociedad Andaluza de Epilepsia 2005: III. Tratamiento antiepiléptico en situaciones especiales. Rev Neurol, 40:683-95.

Serrano Castro PJ, Ramos Lizana J, Pita-Calandre E, et al. 2005. Guía terapéutica en epilepsia de la Sociedad Andaluza de Epilepsia 2005: I. Criterios de inicio y cese y bases farmacológicas del tratamiento antiepiléptico. Rev Neurol, 40:563-71.

Tallis R, Hall G, Craig I, et al. 1991. How common are epileptic disorders in old age? Age Ageing, 20:442-8.

White JR, Walzack TS, Leppik IE, et al. 2003. Discontinuation of levetiracetam because of behavioural side effects: a case control study. Neurology, 61:1218-21. 